\title{
Adaptation of the Structured Clinical Interview for DSM-IV Disorders for assessing depression in women during pregnancy and post-partum across countries and cultures
}

\author{
L. L. GORMAN, M. W. O'HARA, B. FIGUEIREDO, S. HAYES, F. JACQUEMAIN, \\ M. H. KAMMERER, C. M. KLIER, S. ROSI, G. SENEVIRATNE, \\ A.-L. SUTTER-DALLAY and the TCS-PND Group
}

\begin{abstract}
Background To date, no study has used standardised diagnostic assessment procedures to determine whether rates of perinatal depression vary across cultures.
\end{abstract}

Aims To adapt the Structured Clinical Interview for DSM-IV Disorders (SCID) for assessing depression and other nonpsychotic psychiatric illness perinatally and to pilot the instrument in different centres and cultures.

\section{Method Assessments using the adapted SCID and the Edinburgh Postnatal Depression Scale were conducted during the third trimester of pregnancy and at 6 months postpartum with 296 women from ten sites in eight countries. Point prevalence rates during pregnancy and the postnatal period and adjusted 6-month period prevalence rates were computed for caseness, depression and major depression.}

Results The third trimester and 6month point prevalence rates for perinatal depression were $6.9 \%$ and $8.0 \%$,

respectively. Postnatal 6-month period prevalence rates for perinatal depression ranged from $2.1 \%$ to $31.6 \%$ across centres and there were significant differences in these rates between centres.

Conclusions Study findings suggest that the SCID was successfully adapted for this context. Further research on determinants of differences in prevalence of depression across cultures is needed.

Declaration of interest None.
Depressive disorders in the perinatal period occur in women of all cultural and ethnic backgrounds. Empirical studies examining the frequency and causes of postnatal depressive disorders have been the focus of numerous researchers during the past 20 years (O’Hara \& Zekoski, 1988; O’Hara $\&$ Swain, 1996). Studies using standardised diagnostic criteria to assess the prevalence of postnatal depression have primarily been conducted in the USA and Western European countries (Kumar \& Robson, 1984; Watson et al, 1984; O'Hara et al, 1990; Campbell \& Cohn, 1991; Cox et al, 1993). More recently, prevalence studies of postnatal depression have been conducted in non-Western sites such as Japan (Okano et al, 1998; Yoshida et al, 2001), Hong Kong (Lee et al, 2001), South Africa (Cooper et al, 1999) and Dubai (Ghubash \& Abou-Saleh, 1997) with women of more culturally diverse backgrounds.

Prevalence rates for postnatal depression in studies using randomly selected samples of women generally fall between $10 \%$ and $15 \%$. A meta-analysis of 59 studies estimated the average prevalence of postnatal depression to be $13 \%$ (O'Hara \& Swain, 1996). Varying rates across studies were related to the length of the postnatal period being examined and the method of assessment. Higher prevalence rates were significantly associated with longer periods of assessment and the use of self-report instruments, whereas lower rates of postnatal depression were associated with shorter periods of assessment and the use of interview-based measures (O’Hara \& Swain, 1996). Although there have been numerous studies examining the frequency of postnatal depression over the years, none has directly compared rates of antenatal and postnatal depression in women from several different countries and cultural backgrounds within the same

*TCS-PND Group membership and funding detailed in Acknowledgements, p. iv, this supplement. study. Conducting such a multi-site study eliminates potential confounding factors that exist when comparing rates from individual studies, such as the use of different diagnostic measures or systems, or different times of assessment. A multi-site study of postnatal depression also allows examination of the impact of potential psychosocial and cultural influences on rates of the disorder.

The Structured Clinical Interview for DSM-IV Axis I Disorders (SCID-I; First et al, 1994) is a semi-structured diagnostic interview which has been widely used in psychiatric research studies (Spitzer et al, 1992; Williams et al, 1992a), including cross-national epidemiological and treatment studies (Williams et al, 1992b; Weissman et al, 1996). The interview consists of standardised diagnostic questions arranged in modules corresponding to each DSM-IV Axis I disorder (American Psychiatric Association, 1994). The test-retest reliability of SCID-I generally falls between reported values for similar instruments such as the Diagnostic Interview Schedule and the Schedule for Affective Disorders and Schizophrenia (Williams et al, 1992a; First et al, 1996). In an American study using videotaped interviews, paired raters made independent diagnoses of 75 psychiatric out-patients (Riskind et al, 1987). The percentage agreement between raters using the SCID-I was $82 \%(\kappa=0.72)$ for major depressive episode and $86 \%(\kappa=0.79)$ for generalised anxiety disorder.

Reliability studies involving the SCID-I have also been conducted in countries other than the USA. In a Norwegian study, interrater reliability was excellent for the diagnoses of major depressive episode and generalised anxiety disorder with reported $\kappa$ values of 0.93 and 0.95 , respectively (Skre et al, 1991). Similarly, adequate interrater reliability using a Portuguese translation of the SCID-I was demonstrated in a Brazilian study: for major depressive episode, $\kappa=0.88$ (Del-Ben et al, 1996). These two studies demonstrate that the SCID-I can be used reliably with nonAmerican samples. High interrater reliability using the SCID-I and similar structured interviews has also been demonstrated in studies of women in the postnatal period (O'Hara et al, 1990; Wenzel et al, 2001). In an American study examining anxiety and depressive symptoms in women postpartum, interrater agreement for major depressive disorder using the SCID-I was $84 \%, \kappa=0.67$ (Wenzel et al, 2001). 
For the Transcultural Study of Postnatal Depression (TCS-PND), the non-patient research version (SCID-I/NP) was adapted to make it more suitable for use with women in different countries and cultures. Major adaptations included deletion of modules that were not part of the project aims, the addition of new screening questions to the screening module, development of alternative psychosis and mania screening questions, insertion of a module to record the interviewee's smoking history, and replacement of the summary score sheets with a newly developed SCID recording form. Minor adaptations included rewording the original screening questions for alcohol misuse or dependence, substance misuse or dependence and obsessive-compulsive disorder, and revising the overview section to include an expanded section on psychiatric treatment history.

The final modified version used in this study for the assessment of postnatal depression, the SCID-PND, included the following modules: overview; smoking history; screening questions; major depression - current and past; dysthymia; alcohol use disorders; non-alcohol substance use disorders; panic disorder; agoraphobia; social phobia; specific phobia; obsessivecompulsive disorder; post-traumatic stress disorder; acute stress disorder; generalised anxiety disorder; somatisation disorder; anorexia nervosa; bulimia nervosa; and the mood differential module. Lifetime diagnoses of these disorders were assessed at the antenatal interview. At the postnatal interview the diagnostic assessment covered the period between the antenatal and postnatal interviews.

\section{Transcultural Study of Postnatal Depression}

The aim of the TCS-PND was to develop (or modify), translate and validate research instruments that could be used in future studies of postnatal depression in different countries and cultures. The instruments were chosen to assess key aspects of the maternity experience, namely clinical diagnosis, the psychosocial context of pregnancy and motherhood, maternal attachment style, mother-infant interaction, the child's environment, and health service structure, use, and its associated costs. The modified and translated research tools were piloted to test how well they worked in a perinatal setting and in different languages and populations. This paper reports on the development of the SCID-PND and its piloting on women from middle or late pregnancy to about 6 months after delivery.

\section{METHOD}

\section{Study sites}

Ten research centres in eight countries participated in this study: Bordeaux and Paris (France), Dublin (Ireland), Florence (Italy), Iowa City (USA), Keele and London (UK), Porto (Portugal), Vienna (Austria) and Zurich (Switzerland). Details are given by Asten et al (2004, this supplement).

\section{Sample}

Although recruitment methods varied among sites (Asten et al, 2004, this supplement), generally women who were in their second or third trimester of pregnancy were approached at antenatal clinics or childbirth preparation classes and invited to participate in a prospective study examining the psychosocial aspects of postnatal depression. Informed consent was obtained from those interested in participating. Antenatally 296 women were interviewed, 261 of whom also completed a postnatal interview. Demographic characteristics of the sample are described by Asten et al (2004, this supplement).

\section{Procedure}

Participants were first interviewed during the third trimester of pregnancy, on average at 34 weeks' gestation (mean 34.4 weeks, s.d.=3.5) across the ten centres. With the exception of the Zurich group, women were interviewed again, on average, at 26 weeks after delivery (mean 25.8 weeks, s.d.=6.5). Women in Zurich were interviewed at 17 weeks postnatally on average (mean 17.0 weeks, s.d.=1.6).

\section{Other measures}

The Edinburgh Postnatal Depression Scale (EPDS) is a ten-item self-report measure developed to screen and identify women who may be experiencing postnatal depression (Cox et al, 1987). The measure has been used widely in European studies of postnatal depression (Holden et al, 1989; Murray \& Carothers, 1990; Thorpe et al, 1992; Cooper \& Murray, 1994; Augusto et al, 1996; Wickberg \& Hwang, 1996; Appleby et al, 1997; Guedeney \& Fermanian, 1998; Benvenuti et al, 1999) and has been validated for use in postpartum studies of women in several other countries (Boyce et al, 1993; Pen et al, 1994; Holt, 1995; Areias et al, 1996). Although the measure was developed as a screening instrument, a cut-off score of $12 / 13$ identifies women who are most likely to be experiencing post-partum depression (Cox et al, 1987). The measure also has been validated as a screening measure for depression during pregnancy (Murray \& Cox, 1990).

\section{Training}

Training in the use of the adapted SCID-I followed a standard approach that has been used to train clinical interviewers in various epidemiological and intervention studies over the past 20 years (O'Hara et al, 1990, 2000). Research staff from all participating centres underwent at 2-day SCID-PND training workshop held in Keele (UK) in March 1998. Training included an overview of administration guidelines and descriptions of individual modules and symptom criteria for DSMIV Axis I disorders (American Psychiatric Association, 1994). Workshop participants also viewed and rated a videotaped SCID-I interview. All centres were familiar with the user's guide for the SCID-I (First et al, 1994). Subsequent workshops in Paris, the Tyrol, Florence, Manchester and Dublin allotted time to discuss administration and rating questions and issues related to the SCID-PND. No formal reliability analysis was conducted.

\section{Analyses}

Point and adjusted 6-month period prevalence rates were examined for three diagnostic categories of postnatal depression and anxiety and an additional non-diagnostic category that is likely to be indicative of perinatal depression. The broadest diagnostic category, 'caseness', included participants who met DSM-IV criteria for generalised anxiety disorder, minor depressive disorder or major depressive disorder. The second diagnostic category, 'depression', included participants who met DSM-IV criteria for minor or major depressive disorder. The third diagnostic category, 'major depression', included those who met criteria for major depressive disorder only. A final category, 'EPDS 13+', included participants who scored 13 or above on the EPDS. This is the cut-off score found to have the best 
specificity and sensitivity in detecting women who might be experiencing postnatal depression (Cox et al, 1987).

Point prevalence rates were computed for each centre for caseness, depression, major depression and EPDS 13+ at both the antenatal and postnatal assessment points. Period prevalence rates covering the interval between the antenatal and postnatal interviews were computed for caseness, depression and major depression. However, owing to significant variation in the time between interviews among centres (range 21.4-40.4 weeks; $F_{(7,246)}=59.15$, $P<0.001$ ), period prevalence rates were adjusted to estimated 6-month period rates and used in $\chi^{2}$ analyses (or Fisher's exact test when necessary) to compare rates between centres. Because the sample in Keele consisted of only five women, data from the Keele and London centres were combined.

\section{RESULTS}

\section{Antenatal point prevalence rates}

The overall antenatal point prevalence rate for caseness was $11.8 \%$, the rate for depression was $6.9 \%$, the rate for major depression was $3.5 \%$ and the rate for EPDS $13+$ was $8.7 \%$. Rates for each centre are presented in Table 1.

Antenatal point prevalence rates for caseness ranged from $0 \%$ in Vienna to $23.3 \%$ in Bordeaux. The rates of caseness in Bordeaux and Porto were significantly higher than the rate in Vienna $(P=0.048$ and $P=0.032$, respectively), and the rate in Zurich was significantly lower than rates in Porto $(P=0.004)$, Dublin $(P=0.037)$ and Bordeaux $(P=0.011)$. Antenatal point prevalence rates for depression ranged from $0 \%$ in Vienna and Zurich to $19 \%$ in Bordeaux. Rates were significantly lower in Zurich than in Bordeaux $(P=0.004)$, Paris $(P=0.023)$, Porto $(P=0.013)$ and the UK $(P=0.017)$. Although Vienna had no case of depression at the antenatal interview, statistical comparisons with the other centres were not significant, probably because of the small sample sizes. For major depression, antenatal point prevalence rates ranged from $0 \%$ in Vienna, Zurich, Florence and Iowa City to $13.6 \%$ in the UK. The difference in rates was significant between only Zurich and the UK $(P=0.017)$. The point prevalence rates of antenatal depression indicated by a score of $13+$ on the EPDS ranged from

Table I Antenatal point prevalence rates

\begin{tabular}{lcccccc}
\hline Centre & $n$ & $\begin{array}{c}\text { Weeks of } \\
\text { gestation } \\
\text { (Mean) (s.d.) }\end{array}$ & $\begin{array}{c}\text { Caseness (GAD, } \\
\text { minor depression, } \\
\text { MDE) (\%) }\end{array}$ & $\begin{array}{c}\text { Minor or major } \\
\text { depression } \\
(\%)\end{array}$ & $\begin{array}{c}\text { Major } \\
\text { depression } \\
(\%)\end{array}$ & $\begin{array}{c}\text { EPDS I3+1 } \\
(\%)\end{array}$ \\
\hline Vienna & 20 & $36.2(3.7)$ & 0.0 & 0.0 & 0.0 & NA \\
Zurich & 60 & $35.6(2.7)$ & 3.3 & 0.0 & 0.0 & 10.0 \\
lowa City & 35 & $33.8(3.2)$ & 5.7 & 2.9 & 0.0 & 2.5 \\
Florence & 21 & $34.3(1.6)$ & 9.5 & 4.8 & 0.0 & 4.8 \\
Paris & 25 & $33.2(2.0)$ & 12.0 & 12.0 & 8.0 & 12.0 \\
UK & 22 & $31.6(4.8)$ & 13.6 & 13.6 & 13.6 & NA \\
Dublin & 21 & $33.8(4.5)$ & 19.0 & 4.8 & 4.8 & NA \\
Porto & 64 & $34.8(4.5)$ & 20.3 & 10.9 & 4.7 & 9.4 \\
Bordeaux & 21 & $34.3(4.6)$ & 23.8 & 19.0 & 4.8 & 14.3 \\
Total & 289 & $34.4(3.5)$ & 11.8 & 6.9 & 3.5 & 8.7 \\
\hline
\end{tabular}

EPDS, Edinburgh Postnatal Depression Scale; GAD, generalised anxiety disorder; MDE, major depressive episode; NA, not applicable.

I.The EPDS was not administered at the antenatal assessment in Austria and France and was completed by only 2 of 22 women in the UK. Antenatal EPDS data from the UK were not included in analyses because of the small sample size.

Table 2 Postnatal point prevalence rates

\begin{tabular}{lcccccc}
\hline Centre & $n$ & $\begin{array}{c}\text { Weeks of } \\
\text { gestation } \\
\text { (Mean) (s.d.) }\end{array}$ & $\begin{array}{c}\text { Caseness (GAD, } \\
\text { minor depression, } \\
\text { MDE) (\%) }\end{array}$ & $\begin{array}{c}\text { Minor or major } \\
\text { depression } \\
(\%)\end{array}$ & $\begin{array}{c}\text { Major } \\
\text { depression } \\
(\%)\end{array}$ & $\begin{array}{c}\text { EPDS I3+ } \\
(\%)\end{array}$ \\
\hline Vienna & 20 & $27.4(1.8)$ & 0.0 & 0.0 & 0.0 & 5.0 \\
UK & 17 & $30.7(5.1)$ & 0.0 & 0.0 & 0.0 & NA \\
Zurich & 60 & $17.0(1.6)$ & 3.3 & 1.7 & 1.7 & 6.7 \\
Florence & 21 & $27.7(2.2)$ & 4.8 & 0.0 & 0.0 & 9.5 \\
lowa City & 41 & $30.3(4.6)$ & 4.9 & 2.4 & 0.0 & 5.0 \\
Dublin & 17 & $31.1(6.0)$ & 11.8 & 0.0 & 0.0 & 14.3 \\
Paris & 17 & $30.6(2.9)$ & 11.8 & 11.8 & 5.9 & 0.0 \\
Bordeaux & 20 & $27.8(4.7)$ & 15.0 & 15.0 & 10.0 & 0.0 \\
Porto & 48 & $25.8(5.1)$ & 29.2 & 29.2 & 14.6 & 9.5 \\
Total & 261 & $25.8(6.5)$ & 10.0 & 8.0 & 4.2 & 6.6 \\
\hline
\end{tabular}

EPDS, Edinburgh Postnatal Depression Scale; GAD, generalised anxiety disorder; MDE, major depressive episode; NA, not applicable.

$2.5 \%$ in Iowa City to $14.3 \%$ in Bordeaux, with no significant difference between centres.

Overall, the broadest diagnostic, caseness, had a higher point prevalence rate than the other two diagnostic categories, depression and major depression, at the assessment during pregnancy. This is not surprising since caseness included not only diagnoses of major and minor depression, but of generalised anxiety disorder as well. Vienna and Zurich had the lowest rates of antenatal depression and anxiety examined in this study, whereas Bordeaux, Porto and Dublin had the highest rates of antenatal depression and anxiety.

\section{Postnatal point prevalence rates}

The overall postnatal point prevalence rate for caseness was $10.0 \%$, that for depression was $8.0 \%$, that for major depression was $4.2 \%$, and that for EPDS $13+$ was $6.6 \%$. Rates for individual centres are presented in Table 2.

Postnatal point prevalence rates for caseness ranged from $0 \%$ in Vienna and the UK to $29.2 \%$ in Porto. Rates in Porto were significantly higher than those in five of the eight other centres (Vienna, $P=0.007$; UK, $\quad P=0.014 ; \quad$ Zurich, $P<0.001$; Florence, $P=0.027$; Iowa City, $P=0.003)$. Postnatal point prevalence rates 
for depression ranged from $0 \%$ in Vienna, Dublin, Florence and the UK to $29.2 \%$ in Porto. The rate in Porto was significantly higher than that in all the other centres, except Bordeaux and Paris $(P$ range $<0.001-0.014)$. The rate in Bordeaux was also significantly higher than that in Zurich $(P=0.046)$. Current postnatal major depression rates reached a high of $14.6 \%$ in Porto with five of the other eight centres reporting no case of major depression at the time of the postnatal interview. The rate for Porto was significantly higher than rates in other centres only for Zurich and Iowa City ( $P=0.021$ and 0.014 , respectively), probably because of small sample sizes in the other centres. Postnatal point prevalence rates as indicated by EPDS 13+ ranged from $0 \%$ in Bordeaux and Paris to $14.3 \%$ in Dublin.
As with overall rates of caseness, depression and major depression during pregnancy, postnatal point prevalence rates for caseness were higher than the rates for the pure categories of depression and major depression. At the postnatal assessment, it was clear that rates of diagnostic depression and anxiety were highest in Porto and Bordeaux. Ironically, Bordeaux along with Paris had the lowest rates of women scoring $13+$ on the EPDS at post-partum assessment, although both centres recorded a significant number of women meeting DSM-IV criteria for major or minor depression at that interview. A possible explanation for this discrepancy is that the French validation study of the EPDS found lower cut-off scores to be appropriate in community postnatal assessments (Guedeney \& Fermanian, 1998).

\section{Six-month period prevalence rates}

Prevalence rates covering the period between the antenatal and postnatal interviews were adjusted to 6-month period prevalence rates in order to control for the variation in interval between interviews among the different centres. The adjusted 6-month period prevalence rate for each country was calculated by dividing the actual prevalence rate by the average number of weeks between the antenatal and postnatal interview and then multiplying by 26 weeks ( 6 months). The adjusted 6-month prevalence rate was used to calculate the number of cases expected to occur given the sample size of the individual centre, which was then used in $\chi^{2}$ analyses to compare period prevalence rates between centres. Tables 3, 4 and 5 show $\chi^{2}$

Table 3 Six-month period prevalence rates for caseness (generalised anxiety disorder, major and minor depression): chi-squared comparisons

\begin{tabular}{|c|c|c|c|c|c|c|c|c|c|c|c|}
\hline \multirow[t]{2}{*}{ Centre } & \multirow[t]{2}{*}{$n$} & \multirow{2}{*}{$\begin{array}{c}\text { Adjusted } \\
\text { rate (\%) }\end{array}$} & \multicolumn{9}{|c|}{ Centre } \\
\hline & & & Zurich & Florence & Vienna & lowa City & Dublin & UK & Paris & Bordeaux & Porto \\
\hline Zurich & 59 & 6.2 & & & & & & & & & \\
\hline Florence & 21 & 7.3 & 0.034 & & & & & & & & \\
\hline Vienna & 20 & 16.8 & 2.064 & 0.865 & & & & & & & \\
\hline lowa City & 37 & 19.8 & $4.14 I^{*}$ & 1.6 & 0.077 & & & & & & \\
\hline Dublin & 18 & 19.8 & 3.002 & 1.32 & 0.058 & 0 & & & & & \\
\hline UK & 19 & 20.3 & 3.297 & 1.44 & 0.082 & 0.002 & 0.002 & & & & \\
\hline Paris & 17 & 25.8 & $5.353^{*}$ & 2.427 & 0.452 & 0.248 & 0.179 & 0.151 & & & \\
\hline Bordeaux & 21 & 33.9 & $10.187^{* *}$ & $4.517^{*}$ & 1.578 & 1.426 & 0.968 & 0.919 & 0.292 & & \\
\hline Porto & 52 & 38.3 & $17.036 * * *$ & $6.908 * *$ & 3.064 & 3.499 & 2.056 & 2.018 & 0.881 & 0.126 & \\
\hline Total & 264 & 21.7 & & & & & & & & & \\
\hline
\end{tabular}

$* P<0.05 ; * * P<0.01 ; * * * P<0.001$.

Table 4 Six-month period prevalence rates for depression (major and minor): chi-squared comparisons

\begin{tabular}{|c|c|c|c|c|c|c|c|c|c|c|c|}
\hline \multirow[t]{2}{*}{ Centre } & \multirow[t]{2}{*}{$n$} & \multirow{2}{*}{$\begin{array}{c}\text { Adjusted } \\
\text { rate (\%) }\end{array}$} & \multicolumn{9}{|c|}{ Centre } \\
\hline & & & Zurich & Florence & Vienna & Dublin & lowa City & UK & Paris & Porto & Bordeaux \\
\hline Zurich & 59 & 2.1 & & & & & & & & & \\
\hline Florence & 21 & 3.7 & 0.172 & & & & & & & & \\
\hline Vienna & 20 & 16.8 & $5.908 *$ & 1.922 & & & & & & & \\
\hline Dublin & 17 & 16.8 & $5.624 *$ & 1.852 & 0 & & & & & & \\
\hline lowa City & 37 & 17.8 & $7.524 * *$ & 2.394 & 0.01 & 0.008 & & & & & \\
\hline UK & 19 & 20.3 & $7.86 * *$ & 2.681 & 0.082 & 0.075 & 0.053 & & & & \\
\hline Paris & 17 & 25.8 & $10.858 * * *$ & 3.893* & 0.452 & 0.411 & 0.459 & 0.151 & & & \\
\hline Porto & 51 & 30.6 & $17.163^{* * *}$ & $6.107^{*}$ & 1.403 & 1.229 & 1.86 & 0.725 & 0.142 & & \\
\hline Bordeaux & 20 & 31.6 & I5.089*** & $5.563^{*}$ & 1.203 & 1.085 & 1.415 & 0.643 & 0.152 & 0.007 & \\
\hline Total & 261 & 18.3 & & & & & & & & & \\
\hline
\end{tabular}

$* P<0.05 ; * * P<0.01 ; * * * P<0.001$. 
Table 5 Six-month period prevalence rates for major depression: chi-squared comparisons

\begin{tabular}{|c|c|c|c|c|c|c|c|c|c|c|c|}
\hline \multirow[t]{2}{*}{ Centre } & \multirow[t]{2}{*}{$n$} & \multirow{2}{*}{$\begin{array}{l}\text { Adjusted } \\
\text { rate (\%) }\end{array}$} & \multicolumn{9}{|c|}{ Centre } \\
\hline & & & Florence & Zurich & lowa City & Vienna & Dublin & UK & Porto & Paris & Bordeaux \\
\hline Florence & 21 & 0 & & & & & & & & & \\
\hline Zurich & 59 & 2.1 & $0.44 I$ & & & & & & & & \\
\hline lowa City & 36 & 10.2 & 2.281 & 3.009 & & & & & & & \\
\hline Vienna & 20 & 12.6 & 2.81 & 3.648 & 0.074 & & & & & & \\
\hline Dublin & 17 & 16.8 & 3.81 & $5.62 *$ & 0.467 & 0.132 & & & & & \\
\hline UK & 19 & 16.9 & $3.857^{*}$ & 5.898* & 0.515 & 0.147 & 0.0001 & & & & \\
\hline Porto & 50 & 17.3 & $4.142 *$ & $7.638^{*}$ & 0.873 & 0.242 & 0.003 & 0.002 & & & \\
\hline Paris & 17 & 19.7 & $4.538^{*}$ & $7.265^{* *}$ & 0.916 & 0.353 & 0.049 & 0.048 & 0.049 & & \\
\hline Bordeaux & 20 & 19.8 & $4.59^{*}$ & 7.638* & 1.006 & 1.383 & 0.055 & 0.053 & 0.057 & 0.00001 & \\
\hline Total & 259 & 12.3 & & & & & & & & & \\
\hline
\end{tabular}

$* P<0.05 ; * * P<0.01$.

values for adjusted 6-month period prevalence rates for caseness, depression and major depression. The adjusted 6-month period rates reflect cases that occurred during the latter part of pregnancy and the post-partum period, and assume an even distribution of depressive illness across the 26-week period.

The overall 6-month period prevalence rates for caseness, depression and major depression were $21.7 \%, 18.3 \%$ and $12.3 \%$, respectively. These rates are consistent with previously reported rates of depression during pregnancy (Cox et al, 1982; Kumar \& Robson, 1984; O’Hara, 1986; Kitamura et al, 1993) and the post-partum period (O’Hara \& Zekoski, 1988; O’Hara \& Swain, 1996).

Period prevalence rates for caseness ranged from a low in Zurich of $6.2 \%$ to a high in Porto of $38.3 \%$. The Zurich sample contained significantly fewer women meeting criteria for generalised anxiety disorder or minor or major depression than did Iowa City, Bordeaux, Paris or Porto. Florence also had significantly fewer women meeting caseness criteria than did Bordeaux and Porto. Depression period prevalence rates ranged from $2.1 \%$ in Zurich to $31.6 \%$ in Bordeaux. The Zurich rate was significantly lower than that in all other centres except Florence. Florence also had significantly lower rates compared with Bordeaux, Paris and Porto for depression. Period prevalence rates for major depression ranged from $0 \%$ in Florence to $19.8 \%$ in Bordeaux. Rates in Florence and Zurich were significantly lower than those in the UK, Porto, Paris and Bordeaux.
Zurich also had significantly fewer cases of major depression than did Dublin.

Overall, 6-month period prevalence rates revealed lower rates of perinatal depression and anxiety in Florence and Zurich and higher rates in Porto, Bordeaux and Paris. Interestingly, although no woman in the Viennese sample met criteria for current generalised anxiety disorder or depression at the time of the antenatal and postnatal interviews, the period prevalence rate indicates that a high percentage of women $(16.8 \%)$ experienced generalised anxiety disorder and/or major or minor depression during the last part of pregnancy and the early post-partum period.

\section{Other disorders}

Period prevalence rates for other disorders were extremely low, at less than $1 \%$ overall for agoraphobia, anorexia and bulimia nervosa, and $1-2 \%$ for panic disorder and obsessive-compulsive disorder. There was no case of alcohol or substance misuse.

\section{DISCUSSION}

The findings from this pilot study are important in that they suggest that a transcultural comparison of antenatal and postnatal prevalence rates of depression and anxiety using a single measure can be accomplished successfully.

Interestingly, the overall estimated 6-month period prevalence rate of major depression obtained from the current study $(12.3 \%)$ is almost identical to the prevalence rate calculated by O'Hara \& Swain (1996) in a meta-analysis of 59 postpartum depression studies conducted in several countries - European, Western and non-Western - during the past 20 years. The average prevalence rate for postnatal depression obtained in the meta-analysis was $12 \%$ when depression was assessed using standardised interview-based measures. It should be noted that the 6-month prevalence rate obtained in our study reflects not only cases of major depression during the post-partum period, but also cases occurring during the latter part of pregnancy. However, at least $88 \%$ of the women who experienced a major depressive disorder between the antenatal and postnatal interviews reported its onset after delivery.

There were significant differences in prevalence rates of perinatal depression among women participating in the various centres. There may be several explanations for the higher rates of perinatal depression observed in Porto, Paris and Bordeaux and the lower rates found in Zurich and Florence. First, it is possible that these differences occurred by chance, given the relatively small sample sizes in some centres. However, there is evidence that rates of depression and psychiatric illness do vary among countries and cultures. Weissman et al (1996) reported wide variation in lifetime rates of major depression across ten countries participating in a cross-national epidemiological survey (USA, Canada, Puerto Rico, France, West Germany, Italy, Lebanon, Taiwan, Korea and New Zealand). In that study, sample size was 
not a concern as approximately 38000 persons participated.

Another possible explanation for the difference in prevalence rates involves rates of previous episodes of depression among women from the various centres. Previous history of depression is a powerful predictor of perinatal depression (O'Hara et al, 1991). There were significant differences between centres in rates of previous depression, ranging from $10 \%$ or less in Vienna and Zurich to $50 \%$ and over in Iowa City, Paris and the UK. Overall, there were strong associations between previous depression and period prevalence of caseness $\left(\chi^{2}=\right.$ 7.72, $\quad P=0.005), \quad$ depression $\left(\chi^{2}=9.39\right.$, $P=0.002)$ and major depression $\left(\chi^{2}=16.58\right.$, $P<0.001)$. However, when results for individual centres were examined, these associations were significant only in the UK and Vienna, and controlling for past history did not remove the differences between centres. It did not appear, therefore, that different rates of previous depression could explain the higher rates of perinatal depression found in Porto, Paris and Bordeaux, or the lower rates in Zurich and Florence.

Third, there were differences in the demographic characteristics of the sample, notably in terms of social class, with Porto having a much lower proportion of women categorised as 'non-manual class' (43\% compared with $64 \%$ or more elsewhere). 'Manual class' women were more likely to experience perinatal caseness $\left(\chi^{2}=10.91\right.$, $P=0.001)$, depression $\left(\chi^{2}=6.84, P=0.009\right)$ and major depression $\left(\chi^{2}=5.38, P=0.02\right)$, but again, controlling for this variable did not remove the differences between centres.

A fourth hypothesis is that factors related to psychosocial variables associated with depression might have contributed to the higher rates of perinatal depression in Porto, Bordeaux and Paris (Bernazzani et al, 2004, this supplement). Both stressful life events and low levels of emotional support have been associated with postnatal depression (O’Hara \& Swain, 1996). Women in Paris experienced more severe life adversity in the form of acute negative life events and chronic daily stresses during their pregnancy and after delivery compared with women in other centres (Bernazzani et al, 2004, this supplement). Poor emotional support following delivery, another psychosocial variable associated with depression, was reported by a higher percentage of women in Porto than women in other centres (Bernazzani et al, 2004, this supplement).
With regard to the low rates of perinatal depression observed in Florence and Zurich, perhaps psychological factors, such as having a positive attitude towards the pregnancy or having a secure maternal attachment style, buffered women from experiencing depression during this period. Women in both Florence and Zurich reported fewer negative feelings towards the pregnancy than women in other centres (Bernazzani et al, 2004, this supplement). Also, fewer women in Zurich had markedly insecure maternal attachment styles and were perhaps less vulnerable to depression during this transitional period in their life. More insights regarding the validity of these hypotheses can be found in Bernazzani et al (2004, this supplement) and Bifulco et al (2004, this supplement).

Finally, differences in health care systems (see Chisholm et al, 2004, this supplement) may also have contributed to centre differences.

In sum, one of the major aims of the TCS-PND was accomplished in that psychiatric researchers were able to use a standardised, structured diagnostic instrument, the SCID-PND, to assess women from varying cultural backgrounds both during pregnancy and postnatally. Although the overall rate of perinatal depression across centres replicated findings from previous individual studies, cross-cultural comparisons of antenatal and postnatal prevalence rates of depression revealed differences in rates between centres. These findings require verification in larger, multi-centre studies.

\section{REFERENCES}

American Psychiatric Association (1994) Diagnostic and Statistical Manual of Mental Disorders (4th edn) (DSM-IV). Washington, DC: APA

Appleby, L., Warner, R., Whitton, A., et al (1997) A controlled study of fluoxetine and cognitive-behavioural counselling in the treatment of postpartum depression. BMI, 314, 932-936.

Areias, M. E., Kumar, R., Barros, H., et al (1996) Comparative incidence of depression in women and men, during pregnancy and after childbirth. Validation of the Edinburgh Postnatal Depression Scale in Portuguese mothers. British journal of Psychiatry, 169, 30-35.

Asten, P., Marks, M. N., Oates, M. R., et al (2004) Aims, measures, study sites and participant samples of the Transcultural Study of Postnatal Depression. British Journal of Psychiatry, 184 (suppl. 46), s3-s9.

Augusto, A., Kumar, R., Calheiros, J. M., et al (1996) Post-natal depression in an urban area of Porto: comparison of childbearing women and matched controls. Psychological Medicine, 26, |35-|4|.

Benvenuti, P., Ferrara, M., Niccolai, C., et al (1999) The Edinburgh Postnatal Depression Scale: validation for an Italian sample. Journal of Affective Disorders, $\mathbf{5 3}$ |37-|4|.

Bernazzani, O., Conroy, S., Marks, M. N., et ol (2004) Contextual Assessment of the Maternity Experience: development of an instrument for crosscultural research. British Journal of Psychiatry, 184 (suppl. 46), s24-s30.

Bifulco, A., Figueiredo, B., Guedeney, N., et al (2004) Maternal attachment style and depression associated with childbirth: preliminary results from a European and US cross-cultural study. British Journal of Psychiatry, 184 (suppl. 46), s31-s37.

Boyce, P., Stubbs, J. \& Todd, A. (1993) The Edinburgh Postnatal Depression Scale: validation for an Australian sample. Australian and New Zealand Journal of Psychiatry, 27, 472-476.

Campbell, S. B. \& Cohn, J. F. (199I) Prevalence and correlates of postpartum depression in first-time mothers. Journal of Abnormal Psychology, 100, 594-599.

Chisholm, D., Conroy, S., Glangeaud-Freudenthal, N., et al (2004) Health services research into postnatal depression: results from a preliminary cross-cultural study. British Journal of Psychiatry, 184 (suppl. 46), s45-s52.

Cooper, P. J. \& Murray, L. (1994) The impact of psychological treatments of postpartum depression on maternal mood and infant development. In Postpartum Depression and Child Development (eds L. Murray \& P. J Cooper), pp. 20I-220. New York: Guilford.

Cooper, P. J., Tomlinson, M., Swartz, L., et al (1999) Post-partum depression and the mother-infant relationship in a South African peri-urban settlement. British Journal of Psychiatry, 175, 554-558.

Cox, J. L., Connor, Y. \& Kendell, R. E. (1982)

Prospective study of the psychiatric disorders of childbirth. British Journal of Psychiatry, 140, III-117.

Cox, J. L., Holden, J. M. \& Sagovsky, R. (1987)

Detection of postnatal depression. Development of the 10 -item Edinburgh Postnatal Depression Scale. British Journal of Psychiatry, I50, 782-786.

Cox, J. L., Murray, D. \& Chapman, G. (1993) A controlled study of the onset, duration and prevalence of postnatal depression. British Journal of Psychiatry, $\mathbf{1 6 3}$, $27-31$

Del-Ben, C. M., Rodrigues, C. R. C. \& Zuardi, A.W (1996) Reliability of the Portuguese version of the structured clinical interview for DSM-III-R (SCID) in a Brazilian sample of psychiatric outpatients. Brazilian Journal of Medical and Biological Research, 29, 1675-1682.

First, M. B., Spitzer, R. L., Gibbon, M., et al (1994) Structured Clinical Interview for Axis I DSM-IV Disorders. New York: Biometrics Research.

First, M. B., Gibbon, M., Spitzer, R. L., et al (1996) User's Guide for the Structured Interview for DSM-IV Axis I Disorders - Research Version (SCID-I, version 2.0, February 1996 final version). New York: Biometrics Research.

Ghubash, R. \& Abou-Saleh, M.T. (1997) Postpartum psychiatric illness in Arab culture: prevalence and psychosocial correlates. British Journal of Psychiatry, I7I, 65-68.

Guedenay, N. \& Fermanian, J. (1998) Validation study of the French version of the Edinburgh Postnatal Depression Scale (EPDS): new results about use and psychometric properties. European Psychiatry, 13, 83-89.

Holden, J. M., Sagovsky, R. \& Cox, J. L. (1989) Counselling in a general practice setting: controlled study of health visitor intervention in treatment of postnatal depression. BMJ, 298, 223-226. 
Holt, W. J. (1995) The detection of postnatal depression in general practice using the Edinburgh Postnatal Depression Scale. New Zealand Medical Journal, 108 57-59.

Kitamura, T., Shima, S., Sugarwara, M., et al (1993) Psychological and social correlates of the onset of affective disorders among pregnant women. Psychological Medicine, 23, 967-975.

Kumar, R. \& Robson, K. M. (1984) A prospective study of emotional disorders in childbearing women. British Journal of Psychiatry, 144, 35-47.

Lee, D.T. S., Yip, A. S. K., Chiu, H. F. K., et al (200I) A psychiatric epidemiological study of postpartum Chinese women. American Journal of Psychiatry, 158, 220-226.

Murray, L. \& Carothers, A. D. (1990) The validation of the Edinburgh Postnatal Depression Scale on a community sample. British Journal of Psychiatry, 157, 288290

Murray, L. \& Cox, J. L. (1990) Screening for depression during pregnancy with the Edinburgh Depression Scale (EPDS). Journal of Reproduction and Infant Psychology, 8, 99-107.

O'Hara, M. W. (1986) Social support, life events, and depression during pregnancy and the puerperium. Archives of General Psychiatry, 43, 569-573.

O'Hara, M. W. \& Zekoski, E. M. (1988) Postpartum depression: a comprehensive review. In Motherhood and Mental Illness Vol. 2: Causes and Consequences (eds R. Kumar \& I. F. Brockington), pp. 17-63. London: Wright.

O'Hara, M.W., Zekoski, E. M., Philipps, L. H., et al (1990) A controlled prospective study of postpartum mood disorders: comparison of childbearing and nonchildbearing women. Journal of Abnormal Psychology, 99, 3-15

O'Hara, M. W., Schlechte, J. A., Lewis, D. A., et al (199I) Controlled prospective study of postpartum mood disorders: psychological, environmental, and hormonal variables. Journal of Abnormal Psychology, 100 63-73.

O'Hara, M. W. \& Swain, A. M. (1996) Rates and risk of postpartum depression: a meta-analysis. International Review of Psychiatry, 8, 37-54.

O'Hara, M.W., Stuart, S., Gorman, L. L., et al (2000) Efficacy of interpersonal psychotherapy for postpartum depression. Archives of General Psychiatry, 57, 1039-1045.

Okano, T., Nomura, J., Kumar, R., et al (1998) An epidemiological and clinical investigation of postpartum psychiatric illness in Japanese mothers. Journal of Affective Disorders, 48, 233-240.

Pen, T., Wang, L., Jin, Y., et al (1994) The evaluation and application of the Edinburgh Postnatal Depression Scale. Chinese Mental Health Journal, 8, 18-19.

Riskind, J. H., Beck, A. T., Berchick, R. J., et al (1987) Reliability of DSM-III diagnoses for major depression and generalized anxiety disorder using the structured clinical interview for DSM-III. Archives of General Psychiatry, 44, 817-820.

Skre, I., Onstad, W., Togersen, S., et al (199I) High interrater reliability for the structured clinical interview for DSM-III-R Axis I (SCID-I). Acta Psychiatrica Scandinavica, 84, 167-173.

Spitzer, R. L., Williams, J. B.W., Gibbon, M., et al (1992) The structured clinical interview for DSM-III-R (SCID): I. History, rationale, and depression. Archives of General Psychiatry, 49, 624-629.

Thorpe, K. J., Dragonas, T. \& Golding, J. (1992) The effects of psychosocial factors on the mother's emotional well-being during early parenthood: a cross-cultural

\section{CLINICAL IMPLICATIONS}

- Among women from several European countries and the USA, overall rates of depression during pregnancy and the postnatal period (6.9\% and $8.0 \%$, respectively) are significant and deserve clinical attention.

Observed differences in rates of perinatal depression among women from different countries and cultural backgrounds are intriguing and require investigation into the possible contribution of psychosocial factors and variations in health care delivery systems.

- The Structured Clinical Interview for DSM-IV Axis I Disorders is a standardised diagnostic tool with demonstrated utility in cross-cultural research settings and in populations of pregnant and postnatal women.

\section{LIMITATIONS}

- Small samples limit broader generalisations of the findings, pending verification in a large multi-centre study.

- Questions remain regarding the prevalence of antenatal and postnatal depression in women from developing countries and more diverse cultural backgrounds.

- Point prevalence rates reflecting antenatal depression in late pregnancy and postnatal depression at approximately 6 months after delivery, leave open the question of prevalence rates of depression early in pregnancy and up to I year in the postnatal period.

LAURA L. GORMAN, PhD, lowa Depression and Clinical Research Center, University of lowa; MICHAEL W. O'HARA, PhD, Department of Psychology, University of lowa, USA; BARBARA FIGUEIREDO, PhD,

Department of Psychology, University of Minho, Braga, Portugal; SANDRA HAYES, MPsychSc, University College Dublin, Ireland; FRÉDÉRIQUE JACQUEMAIN, PhD, University Department of Child Psychiatry, Centre HospitalierSaint Vincent de Paul, Paris, France; MARTIN H. KAMMERER, Dr med, University of Zurich, Switzerland; CLAUDIA M. KLIER, MD, Department of Psychiatry, University of Vienna, Austria; SIMONE ROSI, MD, Department of Neurologic and Psychiatric Sciences, University of Florence, Italy; GERTRUDE SENEVIRATNE, MRCPsych, Section of Perinatal Psychiatry, Institute of Psychiatry, London, UK; ANNE-LAURE SUTTER-DALLAY, MD, University Department of Psychiatry, Centre Hospitalier Charles Perrens, Bordeaux, France

Correspondence: Professor Michael O'Hara, Department of Psychology, El I Seashore Hall, University of lowa, lowa City, IA 52242, USA. E-mail: mike-ohara@uiowa.edu

study of Britain and Greece. Journal of Reproductive and Infant Psychology, 10, 205-217.

Watson, J. P., Elliott, S. A., Rugg, A. J., et al (1984) Psychiatric disorder in pregancy and the first postnatal year. British Journal of Psychiatry, 144, 453-462.

Weissman, M. M., Bland, R. C., Canino, G. J., et al (1996) Cross-national epidemiology of major depression and bipolar disorder. JAMA, 276, 293-299.

Wenzel, A., Gorman, L. L., O'Hara, M.W., et al (200I) The occurrence of panic and obsessive compulsive symptoms in women with postpartum dysphoria: a prospective study. Archives of Women's Mental Health, 4, 5-12.

Wickberg, B. \& Hwang, C. P. (1996) The Edinburgh Postnatal Depression Scale: validation on a Swedish community sample. Acta Psychiatrica Scandinavica, 94, $181-184$

Williams, J. B. W., Gibbon, M., First, M. B., et al (1992a) The structured clinical interview for DSM-III-R (SCID): II. Multisite test-retest reliability. Archives of General Psychiatry, 49, 630-636.

Williams, J. B.W., Spitzer, R. L. \& Gibbon, M. (1992b) International reliability of a diagnostic intake procedure for panic disorder. American Journal of Psychiatry, 149 560-562.

Yoshida, K., Yamashita, H., Ueda, M., et al (200I) Postnatal depression in Japanese mothers and the reconsideration of 'Satogaeri bunben'. Pediatrics International, 43, 189-193. 